\title{
Automatic Liquid Mixing and Bottle Filling - A Review
}

\author{
Prof. Swapnil R. Kurkute', Mr. Akshay S. Kulkarni ${ }^{2}$, Mr. Mahesh V. Gare ${ }^{3}$, Mr. Soham S. Mundada \\ Department of Electronics \&Telecommunication, SIEM, Nasik, India ${ }^{1,2,3,4}$
}

\begin{abstract}
In today's fast-moving, competitive industrial world, a company must be flexible, cost effective for its survival. In the manufacturing industries there is great demand for industrial automation systems. The industrial automation is necessary in order to streamline operations in terms of acceleration, reliability and system output. In today's economical world, automation plays gradually important role. This is the prototype of "Automatically liquid mixing and bottle filling using PLC \& SCADA" [1]. In our paper "Automatic Liquid mixing and Bottle Filling" is controlled using programmable logic controller (PLC) and SCADA is used for visualizing the system. This paper proposes a mixing and filling management system for industries which is complete application of automation. It's controlling and monitoring makes the system easily accessible and alert the operator in the case of any fault or errors. It is one of the major applications of automation in the soft drink and other beverage industries, where a specific amount of liquid has to be filled continuously into the bottles [2]. The main aim of the paper is to plan, develop and test the real time implementation of PLC, SCADA systems for ratio control based liquid mixing and bottle filling.
\end{abstract}

Keywords: PLC, SCADA, Sensors, Automation, Conveyor.

\section{INTRODUCTION}

This system is designed and developed for "automatic mixing and filling of the liquid into bottles using PLC\&SCADA". The pressure of continuously increase production volumes has stressed older systems and has increased maintenance requirements. For manufacturers, this creates two problems: higher costs and increased production time. Nowadays industries are being challenged to reduce cost, wastage and production time. New technologies are required that will reduce water usage, increase energy efficiency and minimize the production time for beverage industries [3].In order to systematize a liquid mixing plant and minimize human intervention, we are using supervisory control and data acquisition system. The SCADA used for monitoring the plant and helps in reducing the errors caused by humans. For monitoring the internal storage of instruction for the implementing function such as logic, sequencing, timing, counting and arithmetic to control through digital or analog input output modules various types of machines are programmed by PLC (programmable logic controller). The above mentioned two systems are used to monitor and control a plant or equipment in industries such as telecommunications, water and waste control, energy, oil and gas refining and transportation [4].

\section{LITERATURE STUDY}

Dr. A. S. C. S. Sastry, "An automated microcontroller based liquid mixing system". In this paper he concluded that the automated microcontroller-based liquid mixing system provides a very satisfactory performance with a minimal percentage error. The utilization of a microcontroller has been accomplished in the form of the at89s51 microcontroller. The decision to use three microcontrollers was based on the elimination of idle time and the optimization of the mixing process. in addition to this, the utilization of the various proposed components such as dispensers, sensors, pumps, relays, dc motor and an input device was also accomplished[5]. The presented work was published in (IJCSE) international journal on computer science and engineering vol. 02, no. 08, 2010, 2648-2651. Shaukat N. PLC based, "automatic liquid filling process", in this paper he concluded that this application of automation illustrating a PLC based fully automatic untouched liquid filling and mixing system. The system meets the demand of high speed production using the least mechanism requirements. The system has proved to work effectively avoiding unnecessary spill or wastage of liquids. The system also provides high accuracy and precision in proportion of liquid filling and mixing. Although proposed system illustrates the mixing process of two liquids, any number of liquids may be mixed in varying proportions. It is true that the use of PLC is a costly affair particularly for small industries but it offers many advantages that overcome its cost [6]. This paper was published in IEEE multi topic conference, 2002.

Mallaradhya H. M., K. R. Prakash, "Automatic liquid filling to bottles of different height using programmable logic controller. The system has proved to work effectively avoiding unnecessary spill or wastage of liquids. The system also provides high accuracy and precision in proportion of liquids mixed. Although proposed sys- 3 tem illustrates the mixing process of two liquids, any number of liquids maybe mixed in varying proportions. It is true that the use of PLC is a costly affair particularly for small industries but it offers many advantages that overcome its cost. One of the additional features of the proposed system is the use of SCADA that makes it controlled through a remote location. Complete monitoring of the system is possible through SCADA and in fact the process may be stopped or started by SCADA screen. This feature is particularly very useful in case if some fault occurs in the system [7].In proceedings of aece-iraj international conference, July 2013. 


\section{III.METHOD AND ALGORITHMS}

The System has been developed with various controllers such as Microcontroller (AT89s51), PIC microcontroller and PLC and SCADA system. In automatic liquid mixing and bottle filling system PLC and SCADA plays an important role as a processing unit. In review study of this project we are going to study programmable logic controller (PLC) \& Supervisory controlled data acquisition system (SCADA). We are also going to study the Ladder programming.

\subsection{System Controlled using Microcontroller}

The automated microcontroller based liquid mixing system provides a very satisfactory performance with minimal Percentage error. The system consists of 3 microcontrollers for controlling of various sections in the system. The decision tousing 3 microcontrollers were based on elimination of time and optimization of the mixing process [5].

Limitations of Microcontroller

- Microcontroller based system can only be used in small scale industry.

- A single microcontroller cannot control the whole system.

\subsection{System Controlled using PIC}

In PIC based liquid mixing and bottle filling system. PIC Microcontroller plays a vital role. PIC microcontroller is a RISC based Architecture. It is low cost and is used for real time application. The size and cost of the microcontroller are less as compare to PLC and ARM7. The $4 \mathrm{MHz}$ crystal oscillator is used to provide the required clock signals.

Limitations of PIC

- Pre-emptive task switching cannot be implemented.

- Register-bank switching is required to access the entire RAM of many devices.

- Understanding of functionality is quite difficult.

\subsection{System controlled using ARM 7}

In this system LPC2148 (ARM7) plays an vital role of actual processing unit. The $12 \mathrm{MHz}$ crystal oscillator is used to provide the required clock signals to the ARM7. It takes input from power supply of $3.3 \mathrm{~V}$ for its operation this generation introduced the thumb 16 bit Instruction set providing improved code density compared to previous design .it is versatile processor designed for mobile device and other low power electronics. In ARM7 they are combination of $\mathrm{c}$ and assembly language but in ARM7 architecture used more instruction set as compared PIC microcontroller

Limitations of ARM7

- Cost is high.

- Complex instruction set.

- Complicated to designs because number of pin is more.

\subsection{System Controlled using PLC \& SCADA}

Programmable Logic Controller (PLC)

Programmable logic controller is termed as digital computer used for automation of industrial process, such as control of machinery on industry assembly lines. Desperate general- purpose computers, the PLC are designed for multiple inputs and output desperate general purpose computers, the PLC is designed for multiple inputs and output. Arrangements of resistance to vibration and impact, extended temperature ranges, immunity to electrical noise. PLC is programmed to controlled machine operations and is typically stored in the battery backed or non-volatile memory.

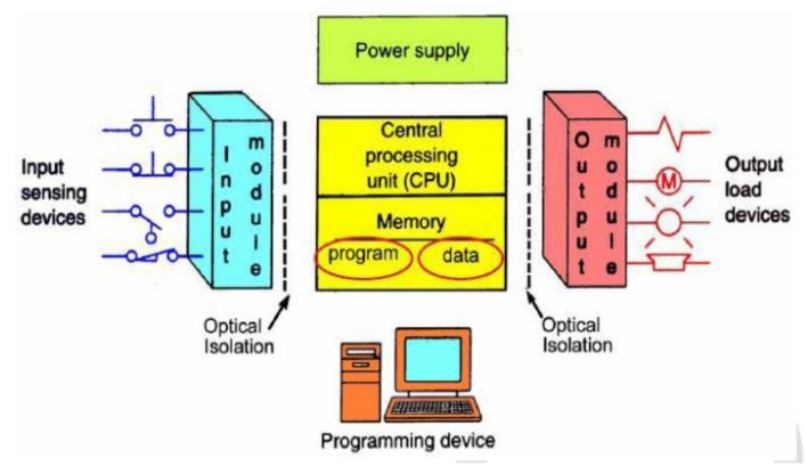

Fig 1 Basic PLC Systems

Merits of System implementation using PLC

- Modular Replacement is possible.

- Easy trouble shooting, Energy saving, and Easy trouble shooting.

- Reduced Space, Greater life and reliability, Error diagnostics programmer.

-The Compatibilities of PLC'S, Logic Control, PID control, Operator control, Signalling and listing, Coordination and communication.

\section{SCADA Systems}

SCADA stands for Supervisory Control and Data Acquisition. As the name indicates, it is not a full control system, but rather focuses on the supervisory level. What is SCADA? It is used to monitor and control plant or equipment. The control may be automatic or initiated by operator commands. The data acquisition is accomplished firstly by the RTU's scanning the field inputs connected to the RTU (it may be also called a PLC- Programmable logic controller.). This is usually at a fast rate [8].

Merits of SCADA system:

- The computer can record and store a very large amount of data.

- The data can be displayed in any way the user requires.

- Thousands of sensors over a wide area can be connected to the system.

- The operator can incorporate real data simulations into the system.

- Many types of data can be collected from the RTUs.

- The data can be viewed from anywhere, not just on site.

\section{IV.EXPECTED RESULT}

The system for automatic liquid mixing and bottle filing system should have low power consumption, low project cost, flexibility and high accuracy. At the same time it should deliver specific amount of liquid to be filled in bottles for saving the operational time. The important expectation from such system is the system should be fully automated i.e. system should be capable to fill the bottles 
according to the amount of liquid by sensing the size of bottle. It should be capable to fill more than one bottle at a time of different size. The system should be flexible not only for different liquid mixing but also for the solids. The system should be capable to monitor the parameters such as temperature, liquid level, quantity, presence of bottle etc.

\section{CONCLUSION}

This paper presents different types of techniques used for Automatic liquid mixing and bottle filling system. In this paper we have a literature study of implantation such system using PIC controller, ARM7, Microcontroller, PLC and SCADA. After comparative study of above techniques we found that PIC controller have drawback that Register-bank switching is required to access the entire RAM of many devices. We can implement system using ARM7 but it is having Complications to designs as number of pin is more. We cannot control whole process using single microcontroller hence it is useful for small project implementations. Hence system should tread off all the above requirements which are possible if the system is implemented using PLC\& SCADA. System implemented using PLC \& SCADA overcomes all the drawbacks and will give efficient output as per requirements.

\section{ACKNOWLEDGMENT}

We would like to express profound gratitude to Dr. R. G. Tated (Principal, SIEM Nasik) for his valuable support, encouragement, supervision and useful suggestions throughout this work. Also to Prof. D. P. Patil, Prof. B.D. Deore, Prof. P.P. Chaudhari and Prof. A. R. Chordiya of department of Electronics \& Telecommunication for moral support and continuous guidance enabled us to complete this work successfully.

\section{REFERENCES}

[1] Jagat Diman,, Dilip Kumar," Hybrid method for automatically filling of the chemical liquid into bottles using plc and scada", IJERGS, Vol 2, issue - 6,OCT-NOV 2014(ISSN 2091-2730).

[2] Hemant Ahuja, Arika Singh," Automatic filling management system for industry" International jornal of immerging technology and advanced engineering (ISSN 2250-2459), volume 4,feb-2014.

[3] Mihir Panchal, AashishPanaskar, Prof. Lalit Kumar,"PLC Based Liquid Filling and Mixing"International Journal of Electrical and Electronics Research ISSN 2348-6988 (online) Vol. 3, Issue 1, pp: (249-253), Month: January - March 2015

[4] S.T.Sanamdikar andVartak C "Color making and mixing process using PLC" International Journal of Emerging Trends \& Technology in Computer Science (IJETTCS)Volume 2, Issue 5, September - October 2013.

[5] Dr. A. S. C. S. Sastry,K.N.H.Srinivas, Ch V S R G Krishna, Ch.SeshaKiranKumar "An Automated Microcontroller Based Liquid Mixing System", (IJCSE) International Journal on Computer Science and Engineering,Vol. 02, No. 08, 2010, 2648-265

[6] Shaukat. N, PLC based automatic liquid filling process, multitopic conference2002, IEEE publication.

[7] Mallaradhya H. M., K. R. Prakash, Automatic Liquid Filling to Bottles of Different Height Using Programmable Logic Controller

[8] Hillebrand, Cary, Expert Three, Technical expert specializing in the planning and design of SCADA based and Distributed control system.

\section{BIOGRAPHIES}

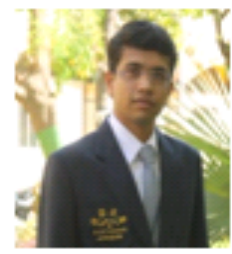

Prof. Swapnil R. Kurkute - Received $\mathrm{BE}$ in Electronics and Communication Engineering and $\mathrm{ME}$ in Communication from North Maharashtra University, JalgaonMaharashtra. Presently working as a Assistant Professor in department of Electronics and Telecommunication Engineering of sandip foundation's SIEM, Nasik.

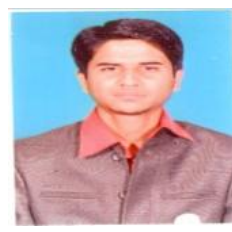

Mr. Akshay Kulkarni - PursuingBE in Electronics and Telecommunication engineering from department of Electronics and Communication Engineering of sandip foundation's SIEM, Nasik.

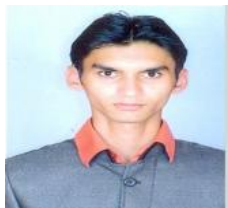

Mr. Soham Mundada - PursuingBE in Electronics and Telecommunication engineering from department of Electronics and Communication Engineering of sandip foundation's SIEM, Nasik.

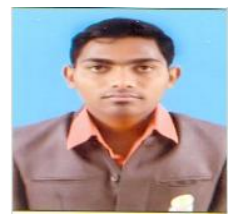

Mr. Mahesh Gare -PursuingBE in Electronics and Telecommunication engineering from department of Electronics and Communication Engineering of sandip foundation's SIEM, Nasik. 